\title{
Acoustic Nonlinearity of Surface Wave in a Fatigued Aluminum Alloy Specimen
}

\author{
Kyung-Young Jhang ${ }^{1}$, Jaeik Lee ${ }^{2, * 1}$ and Taehun Lee ${ }^{2, * 2}$ \\ ${ }^{1}$ School of Mechanical Engineering, Hanyang University, Haengdang-dong 17, Seongdong-gu, Seoul 133-791, Korea \\ ${ }^{2}$ Automotive Engineering, Graduate School of Hanyang University, Haengdang-dong 17, Seongdong-gu, Seoul 133-791, Korea
}

\begin{abstract}
This paper reports a case study for the evaluation of surface fatigue damage by using the acoustic nonlinearity of surface waves. A measurement system using contact angle beam transducers was constructed to measure the acoustic nonlinearity of surface waves based on the harmonic measurement. In order to prove the general properties of acoustic nonlinearity, dependencies of the second harmonic amplitude on the fundamental frequency amplitude and the propagation distance were tested first, where the effect of frequency-dependent attenuation was compensated for. The results showed good agreement with the theoretical expectations. Next, an aluminum 6061 T6 specimen, of which the surface was damaged by a three-point bending fatigue test, was experimented on and magnitudes of nonlinear parameter, measured before and after the fatigue test, were compared. We could see a clear increase in the nonlinear parameter after the fatigue test at the central position of the specimen, where the surface fatigue damage was expected to be concentrated. [doi:10.2320/matertrans.I-M2011846]
\end{abstract}

(Received November 11, 2009; Accepted March 15, 2011; Published January 18, 2012)

Keywords: surface wave, acoustic nonlinearity, harmonic generation, bending fatigue, surface damage

\section{Introduction}

Most of the fracturing in material is initiated from the surface, so that it is important to evaluate the surface degradation for the prevention of fracture. Recently, the acoustic nonlinearity of surface waves has been considered an effective method to evaluate the degradation on the surface of material. ${ }^{1)}$

Basic characteristics of the acoustic nonlinearity in surface waves has been known from former researches, ${ }^{2-5)}$ and it has been found to be similar to the acoustic nonlinearity in longitudinal waves. That is, the second harmonic wave is generated during the propagation of surface waves and its amplitude is proportional to the power of the fundamental frequency component and the propagating distance. There have been many experiments and theoretical accesses to verify that there are obvious correlations between the acoustic nonlinearity of surface waves and surface damage ${ }^{6-9)}$ Now we need more studies on practical applications to various surface damages.

This paper reports a feasibility study to apply the acoustic nonlinearity of surface waves to the evaluation of surface damage caused by bending fatigue. A measurement system using contact angle beam transducers was constructed to measure the acoustic nonlinearity of surface waves. The test specimen was an Aluminum 6061 alloy block. In order to prove the basic characteristics of the acoustic nonlinearity, dependencies of the second harmonic amplitude on the fundamental frequency amplitude and the propagation distance were tested first. Particularly in the analysis of dependency on the propagation distance, the effect of frequency-dependent attenuation was considered compensatory to the difference in the attenuation of both the primary wave and the harmonic wave.

\footnotetext{
${ }^{* 1}$ Graduate Student, Graduate School of Hanyang University. Present address: Samsung Engineering Co., Ltd. Seoul 135-708, Korea

${ }^{* 2}$ Graduate Student, Graduate School of Hanyang University. Present address: Central Research Institute, Korea Hydro and Nuclear Power Co., Ltd. Daejeon 305-343, Korea
}

Next, in order to demonstrate the posibillity of surface damage evaluation by the acoustic nonlinearity of surface waves, the specimen, of which the surface was damaged by a three-point bending fatigue test, was experimented on and magnitudes of nonlinear parameters measured before and after the fatigue test, were compared.

\section{Theoretical Background}

\subsection{The acoustic nonlinearity of surface waves}

A surface wave is a synthesized wave composed of longitudinal and transverse components. When the medium is isotropic and has weak acoustic nonlinearity, the nonlinearity of the transverse wave can be ignored because of the thirdorder elastic modulus symmetry. ${ }^{4)}$ Therefore, only the longitudinal wave component is considered for surface wave nonlinearity. Thus, the acoustic nonlinearity of surface waves has similar characteristics to the nonlinearity of longitudinal waves, and the amplitude of the second harmonic wave generated after wave propagation is given as eq. (1). ${ }^{1)}$

$$
A_{2} \propto\left(\beta k x A_{1}^{2}\right) / 8
$$

where $\beta$ is the nonlinear parameter, $A_{1}$ and $A_{2}$ are the amplitudes of the primary wave and the secondary harmonic wave, $k$ is the wave number, and $x$ is the propagation distance.

Thus, the nonlinear parameter of surface waves can be estimated by measuring the amplitudes of the primary wave and the secondary harmonic wave, when both the wave number and the propagation distance are known.

In this study however, we measure the parameter $\beta^{\prime}$ defined as eq. (2), which is convenient in a relative comparison when the wave number and the propagation distance are fixed.

$$
\beta^{\prime}=A_{2} / A_{1}^{2} \propto(\beta k x) / 8
$$

The magnitude of this parameter varies with material degradation, so we can then evaluate the grade of degradation by monitoring this parameter. However, when the propaga- 
tion distance is not constant, it should be noted that $\beta^{\prime}$ is in proportion to the propagation distance, $x$.

\subsection{The compensation of frequency-dependent attenu- ation}

In the derivation of eq. (1), it is assumed that there is no frequency-dependent attenuation. However, the attenuation of normal surface waves is frequency-dependent, and therefore attenuations of the primary wave and the harmonic wave are different. This causes an error in the nonlinear parameter estimated by eq. (2) using the measured amplitudes of the primary wave and the secondary harmonic wave. Due to this error, the compensation of such frequency-dependent attenuation is needed when measuring the amplitudes of the primary wave and the secondary harmonic wave.

Normal surface wave attenuation can be introduced by employing energy losses arising from exponential damping of the form

$$
A^{\prime}=A \exp (-\alpha x)
$$

where, $A$ is the initial amplitude, $A^{\prime}$ is the attenuated amplitude measured after the propagation of distance $x$, and $\alpha$ is the attenuation coefficient.

Thus, the amplitude at the fundamental frequency before being attenuated can be obtained by

$$
A_{1}=A_{1}^{\prime} / \exp \left(-\alpha_{1} x\right)
$$

where $A_{1}{ }^{\prime}$ is the measured amplitude at the fundamental frequency and $\alpha_{1}$ is the attenuation coefficient at the fundamental frequency.

While for a surface wave which experiences normal exponential attenuation and exhibits no significant nonlinear attenuation, the amplitude of the second harmonic can be represented as eq. (5). ${ }^{10,11)}$

$$
A_{2}{ }^{\prime}=\left(\beta_{0} A_{1}^{2}\right)\left\{\exp \left(-2 \alpha_{1} x\right)-\exp \left(-\alpha_{2} x\right)\right\} /\left\{\left(\alpha_{2}-2 \alpha_{1}\right)\right\} \text {. }
$$

$\beta_{0}=\beta k^{2} / 8$, and $\alpha_{2}$ is the attenuation coefficient at the second-harmonic frequency.

Thus, the amplitude at the second-harmonic frequency before being attenuated can be obtained by

$$
A_{2}=\left\{\left(\alpha_{2}-2 \alpha_{1}\right) x A_{2}{ }^{\prime}\right\} /\left\{\exp \left(-2 \alpha_{1} x\right)-\exp \left(-\alpha_{2} x\right)\right\},
$$

where $A_{2}{ }^{\prime}$ is the measured amplitude at the second-harmonic frequency. Finally, the parameter $\beta^{\prime}$ is estimated using these compensated amplitudes.

\section{Experimental Procedure}

\subsection{Experimental setup and specimens}

Figure 1 shows a schematic diagram of the measurement system. Here, the signal from the function generator is amplified in a gated amplifier, (Ritec GA2500) and derives a transmitting transducer to launch a surface wave on the surface of the specimen, whileanother transducer receives the propagated surface wave. After amplification in the pulserreceiver (Panametric 500PR), the received signal was monitored on the digital oscilloscope and analyzed on a computer for the final frequency analysis. We used Panametics wedge-transducer assemblies to transmit and receive the surface wave and the wedge angle was $66^{\circ}$.

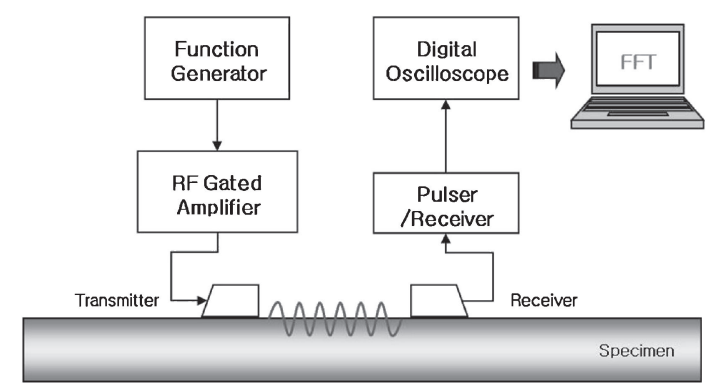

Fig. 1 Schematic diagram of measurement system.

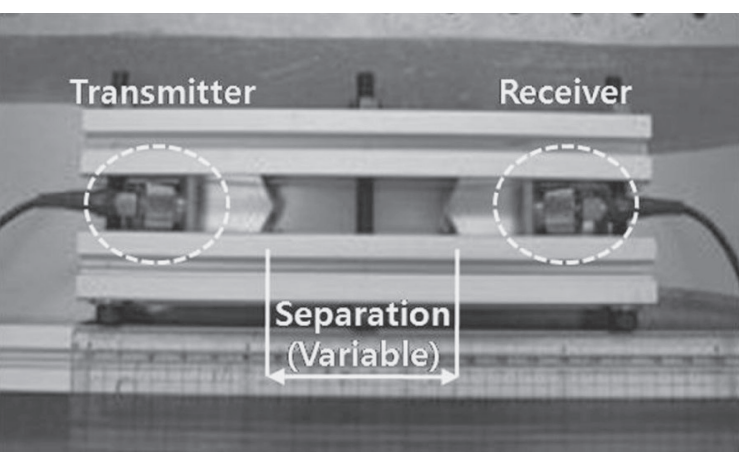

Fig. 2 Experimental jig to hold transducers.

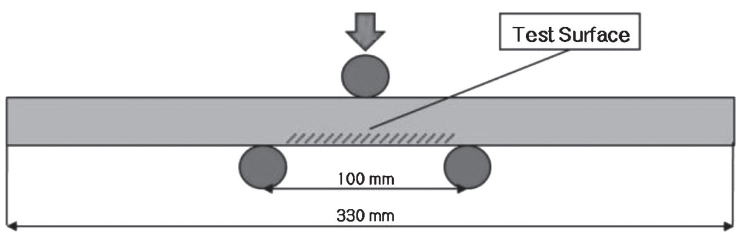

Fig. 3 Three-point bending test and the surface tested for the application of the acoustic nonlinearity of surface wave to the surface fatigued damage.

Figure 2 shows a jig to hold the transducers in a fixed separation.

Al6061 T6 thick blocks were used as specimens. Two sizes of specimen were prepared; $150 \mathrm{~mm} \times 600 \mathrm{~mm} \times 50 \mathrm{~mm}$ and $30 \mathrm{~mm} \times 330 \mathrm{~mm} \times 20 \mathrm{~mm}$. The larger block was used for the general test and the smaller block was used for the fatigue test.

\subsection{The three-point bending fatigue test}

In order to fabricate a specimen damaged on the surface, we carried out a three-point bending fatigue test as shown in Fig. 3. $13 \mathrm{kN}$ was loaded at the central position of the specimen by a loading roller and the opposite side of the specimen was supported by two fixed rollers. The stress ratio was 0.1 and the loading cycle was $10^{7}$ cycles. Note that the $10^{7}$ cycles of loading is approaching the beginning of external deformation. Only the opposite side of the loading surface was tested because the loading surface was damaged by contact with the loading roller.

Figure 4 shows the result of a numerical analysis of the equivalent alternating stress on the test surface under the given loading condition. We can see the stress is concentrated on the central region of the test surface. From this, we can expect that the fatigue damage is concentrated in this region also. 


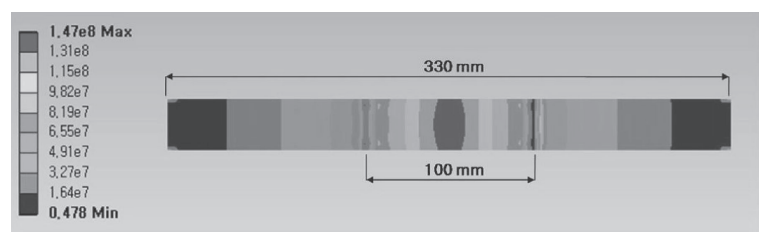

Fig. 4 Results of numerical analysis for the equivalent alternating stress on the test surface of specimen under 3-point bending fatigue condition.

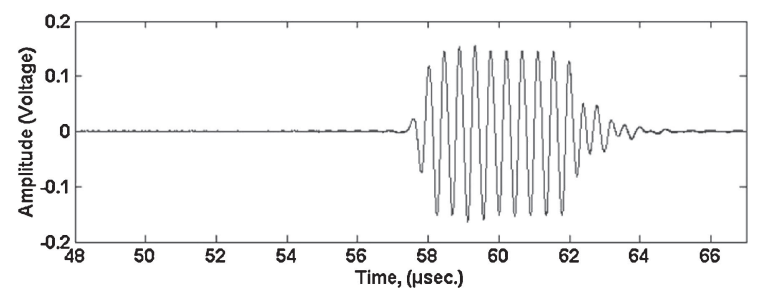

Fig. 5 Sample signal of received waveform.

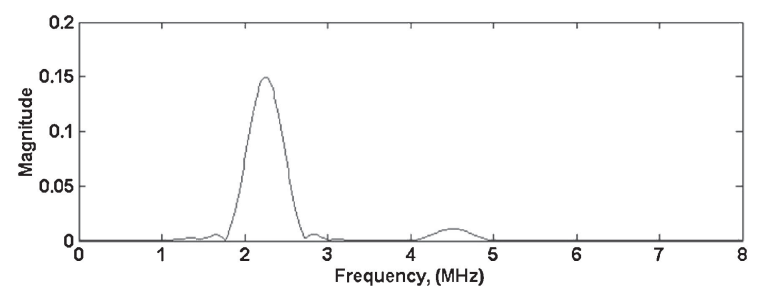

Fig. 6 Magnitude spectrum of signal shown in Fig. 5.

\section{Results}

\subsection{The general test for the acoustic nonlinearity of surface waves}

In order to prove the general properties of the acoustic nonlinearity of surface waves, dependencies of the second harmonic amplitude on the fundamental frequency amplitude and the propagation distance were tested first.

Figures 5 and 6 show a sample of the received signal and its spectrum, respectively. In this test, a $2.25 \mathrm{MHz}$ transducer was used as a transmitter and a $5 \mathrm{MHz}$ transducer was used as a receiver. Since the amplitude of the second harmonic frequency is much smaller than the amplitude of fundamental frequency, the receiving transducer having the center frequency near the second harmonic frequency and narrow bandwidth was utilized to detect the the second harmonic components sensitively. A high voltage $2.25 \mathrm{MHz}$ toneburst signal with 10 cycles was used for transmitting the waveform. It is known that narrow band signal is advantageous to detect the higher harmonic amplitude effectively. ${ }^{12)}$ In Fig. 6, we can confirm that both the fundamental frequency and its double frequency for the second harmonic wave are being detected well. In this experiments, since transducers, wedges, imperfect acoustic coupling and measurement system also may generate extra 2nd harmonic component, we have paid attention to minimize the effect of these sources of extra 2 nd harmonics and to hold this extra component in constant by keeping all experimental conditions in consistent.

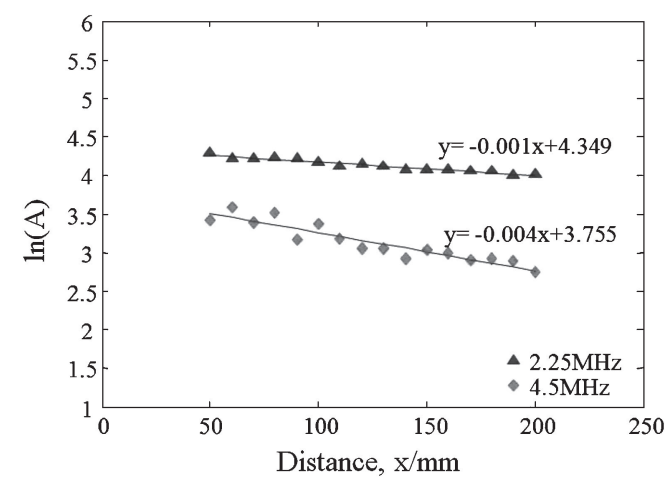

Fig. 7 Estimation of attenuation coefficients at the fundamental and second-harmonic frequency.

Next, in order to compensate for the effect of frequencydependent attenuation, we obtained attenuation coefficients $\alpha_{1}$ and $\alpha_{2}$ at the fundamental and second-harmonic frequencies for the virgin-state specimen by monitoring the change in the amplitude of the received signal when the propagation distance varies. The receiving transducer was fixed and the transmitting transducer was moved to change the propagation distance. Figure 7 shows the result in a semi$\log$ scale. In this experiment, a $2.25-5 \mathrm{MHz}$ transducer combination was used, but a $2.25 \mathrm{MHz}$ wave was transmitted and received for measuring $\alpha_{1}$, and a $4.5 \mathrm{MHz}$ wave was transmitted and received for measuring $\alpha_{2}$. The input power was same in both cases. The slope of the fitted line corresponds to the attenuation coefficient. Since rectangular transducer was used as a line source so that the diffraction effect was regarded as small, the influence of beam spread by diffraction was not taken into account. Obtained values of $\alpha_{1}$ and $\alpha_{2}$ are 0.001 and $0.004 \mathrm{~mm}^{-1}$, respectively. These agree with the other researcher's report that the attenuation coefficient of surface waves is proportional to the square of the frequency. ${ }^{13)}$

We have carried out two experiments to prove that the measured nonlinearity reflects the actual acoustic nonlinearity of surface waves and coinsides with the theoretical tendencies: One was the dependency of the second harmonic amplitude on the fundamental frequency amplitude, and the other is the dependency of parameter $\beta^{\prime}$ on the propagation distance. From the theoretical background, the second harmonic amplitude would be linearly proportional to the square of the fundamental amplitude as shown in eq. (1), which would mean that parameter $\beta^{\prime}$ would be constant regardless of the variation of the fundamental frequency amplitude when the wave number and the propagation distance are fixed. However, when the propagation distance varies, the parameter $\beta^{\prime}$ would be linearly proportional to the propagation distance, as shown in eq. (2).

Figure 8 shows the result of the relationship between $A_{1}{ }^{2}$ and $A_{2}$, when the input signal amplitude is increased. The measurement was made twice under the same conditions. The result shows the linearly proportional relationship and good agreement with the above theoretical expectation.

Figure 9 shows the result of the dependency of parameter $\beta^{\prime}$ on the propagation distance. The measurement was made twice for the virgin-state specimen, while the propagation distance was changing from 80 to $150 \mathrm{~mm}$ in $10 \mathrm{~mm}$ 


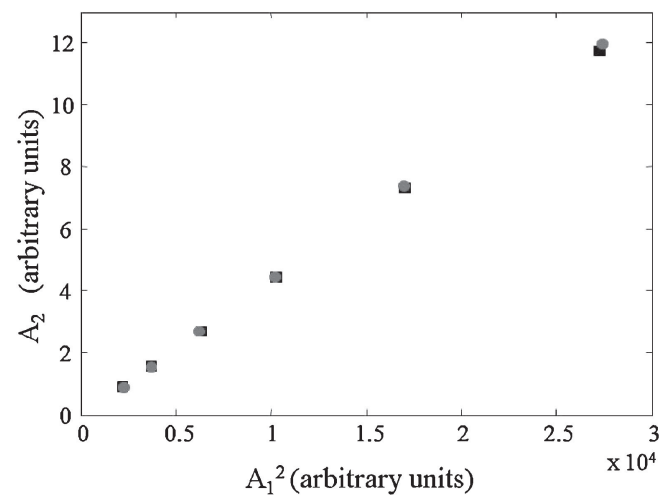

Fig. 8 Experimental result for the dependency of the second harmonic amplitude on the fundamental frequency amplitude: $A_{1}^{2}$ vs. $A_{2}$.
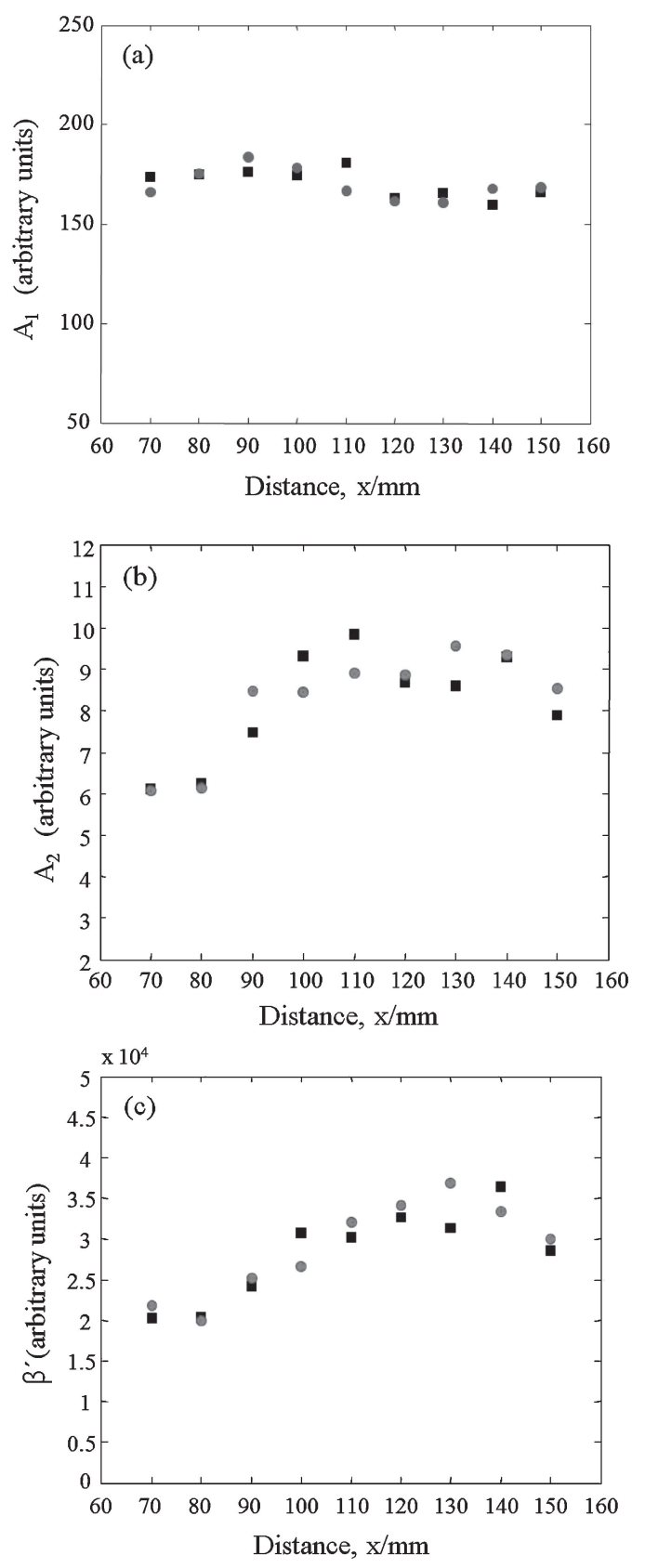

Fig. 9 Experimental results for the dependency on the propagation distance of (a) $A_{1}$, (b) $A_{2}$, and (c) $\beta^{\prime}$. increments. Figures 9(a) and 9(b) show the amplitudes at the fundamental and second harmonic frequencies. We can see that the amplitude at the fundamental frequency $A_{1}$ is almost constant, which means that the compensation of attenuation was adoptable. The amplitude at the second harmonic frequency $A_{2}$ increases proportionally to the propagation distance. This is also identical to the theoretical expectation as described in eq. (1). In addition, since the extra 2nd harmonics generated by experimental system described in the above will not be accumulated with the propagation distance, we can regard the propagation distance dependent 2nd harmonics measured here is mostly from the nonlineaity of material. Figure 9(c) shows $\beta^{\prime}$. We can see that $\beta^{\prime}$ is linearly proportional to the propagation distance, and this agrees well with the theoretical expectation from eq. (2). From these results, we can prove that the measured nonlinearity reflects the actual acoustic nonlinearity of surface wave and coinsides with the theoretical tendencies.

However, in far distance, the effect of intensity reduction by beam spreading and energy leak into the air may not be negligible, and therefore the amplitude of A2 and relative nonlinear parameter were under-compensated.

\subsection{The correlation between parameter $\beta^{\prime}$ and surface fatigue damage}

To demonstrate the applicability of the nonlinear surface wave to the evaluation of surface damage, we measured the nonlinear parameter $\beta^{\prime}$ on the surface of the fatigued specimen. In this experiment, a $1.5 \mathrm{MHz}$ transducer was used as a transmitter and a $3.5 \mathrm{MHz}$ transducer was used as a receiver, which were smaller than the transducers used in the previous tests and as such, more suitable to attach to the smaller specimen. The separation of transducers shown in Fig. 2 was fixed at $20 \mathrm{~mm}$.

The measuring position was moved from the center of the specimen, both to the left and right sides, in $20 \mathrm{~mm}$ increments. The total range of measurement was $60 \mathrm{~mm}$ from the center to both sides, and was repeated twice. For the comparison, the same measurements were made on the intact specimen before the fatigue test.

The results are shown in Fig. 10 by the relative ratio of nonlinear parameter $\beta^{\prime} / \beta_{0}^{\prime}$, where $\beta_{0}^{\prime}$ is the mean value of the nonlinearity parameter obtained from the intact specimen. We can see a clear increase in the nonlinear parameter at the central position of the fatigued specimen. The overall pattern of distribution agrees well with the the results of the numerical analysis shown in Fig. 4. At the same time, the nonlinear parameter remains almost constant in the intact specimen, regardless of the position. Comparing the results from the fatigued specimen with those of the intact specimen, a clear increase in the nonlinear parameter was noticed after the fatigue test at the central position of the specimen, where the surface fatigue damage was expected to be concentrated. Cyclic loading in metals usually generates large numbers of dislocations. The increase of the dislocation density results in the increase of nonlinear parameter due to the evolution of lattice distortion. In this result, the dislocation density might be a dominant factor influencing the ultrasonic nonlinearity. From this, we can say that the nonlinear parameter has a strong correlation with the surface degradation. 


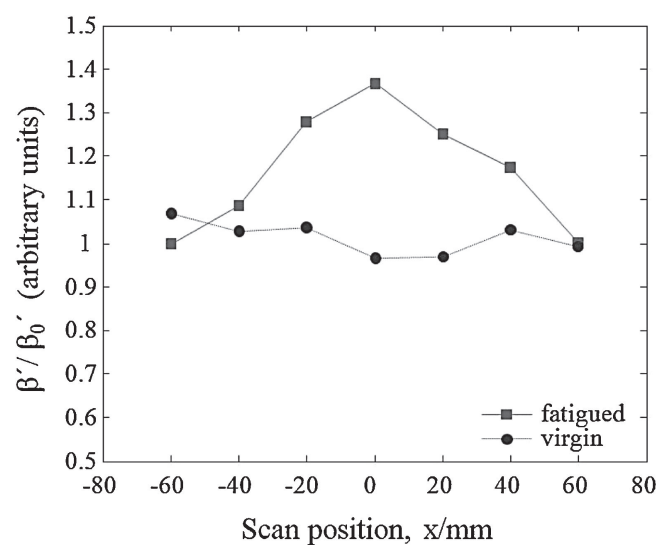

Fig. 10 Comparison of nonlinear parameter measured before and after the fatigue test.

Note that the measuring point corresponds to the center of the transducer separation, so that the measured magnitude at a point is the mean value of the acoustic nonlinearity over the region between the two transducers. Therefore, if we want to localize the acoustic nonlinearity in more detail — that is, if we want to increase the spatial resolution - then we have to shorten the separation between the transducers. However, the separation of transducers will be limited by the sensibility of the measurement system to measure the nonlinear parameter, as the second harmonic amplitude is dependent on the propagation distance.

\section{Conclusion}

In this paper, a case study of the evaluation of surface fatigue damage by using the acoustic nonlinearity of surface waves was reported. A measurement system using contact angle beam transducers was constructed to measure the acoustic nonlinearity of surface waves. The effect of frequency-dependent attenuation was introduced to compensate for the different attenuations in the measured amplitudes at both the fundamental and second-harmonic frequencies.

The experiment, which set out to prove the general properties of the acoustic nonlinearity of surface waves, resulted in showing that the second harmonic amplitude was linearly dependent on the square of the fundamental frequency amplitude and that the measured nonlinear parameter $\beta^{\prime}$ was linearly proportional to the propagation distance. These were all in good agreement with the theoretical expectations, and we could verify the usefulness of our measurement method to measure the acoustic nonlinearity of surface waves.

Next, an aluminum 6061 T6 specimen, of which the surface was damaged by a three-point bending fatigue test, was experimented on to demonstrate the applicability of nonlinear surface waves to the evaluation of surface damage. Experimental results showed a clear increase in the nonlinear parameter after the fatigue test at the central position of the specimen, where the surface fatigue damage was expected to be concentrated.

From these results, we can confirm that the acoustic nonlinearity of surface waves has a strong correlation with the surface degradation and is possibly applicable in the evaluation of surface damage.

\section{Acknowledgements}

This work was financially supported by National Research Foundation of Korea (KRF-2007-313-D00010 and 20100018195).

\section{REFERENCES}

1) K. Y. Jhang: Int. J. Precision Eng. Manufact. 10 (2009) 123-135.

2) J. Herrmann, J. Y. Kim, L. J. Jacobs, J. Qu, J. W. Littles and M. F. Savage: J. Appl. Phys. 99 (2006) 124913 1-8

3) J. L. Rose: Ultrasonic Waves in Solid Media, (Cambridge University Press, USA, 1999) pp. 44-49.

4) J. L. Rose: Ultrasonic Waves in Solid Media, (Cambridge University Press, USA, 1999) pp. 90-92.

5) J. Y. Kim, L. J. Jacobs and J. Qu: J. Acoustical Soc. Am. 120 (2006) 1266-1273.

6) D. J. Barnard, L. J. H. Brasche, D. Raulerson and A. D. Detyar: Rev. Quantitative Nondestruct. Eval. 22 (2003) 1393-1400.

7) G. Shui, J. Y. Kim, J. Qu, Y. S. Wang and L. J. Jacobs: NDT\&E Int. (2008) 326-329.

8) S. P. Sagar, A. Metya, N. Parida and R. N. Ghosh: Rev. Quantitative Nondestruct. Eval. 27 (2008) 1260-1266.

9) H. Ogi, M. Hirao and S. Aoki: J. Appl. Phys. 90 (2001) 438-442.

10) P. J. Vella, T. C. Padmore and G. I. Stegeman: J. Appl. Phys. 45 (1974) 1993-2006.

11) J. Lee, G. Kwon, T. Lee and K. Y. Jhang: J. Korean Soc. Nondestruct. Testing 29 (2009) 344-350.

12) Y. Tsukahara: Appl. Phys. Lett. 59 (1991) 2384-2385.

13) C. Campbell: Surface Acoustic Wave Devices and Their Signal Processing Application, (Academic Press, USA, 1989) pp. 123-124. 